\title{
Measurements of intrinsic emittance dependence on rf field for copper photocathodes
}

\author{
Eduard Prat, ${ }^{*}$ Simona Bettoni, Hans-Heinrich Braun, Marta Csatari Divall, \\ and Thomas Schietinger \\ Paul Scherrer Institut, CH-5232 Villigen PSI, Switzerland \\ (Received 1 April 2015; published 22 June 2015)
}

\begin{abstract}
Radio-frequency (rf) photoinjectors are used to generate high-brightness electron beams for a wide range of applications. Because of their outstanding beam quality, they are particularly well-suited as sources for $\mathrm{X}$-ray free-electron lasers (FELs). The beam emittance, which is significantly influenced by the intrinsic emittance of the cathode, is fundamental for FELs, since it has a strong impact on the lasing performance and it defines the length and cost of the facility. In this paper we present measurements of the intrinsic emittance as a function of the rf field for a copper photocathode. Our measurements match with the theoretical expectations, showing that the intrinsic emittance can be reduced by decreasing the rf field at the cathode. We obtained normalized intrinsic emittances down to $350 \mathrm{~nm} / \mathrm{mm}$, the lowest values ever measured in a rf photoinjector.
\end{abstract}

DOI: 10.1103/PhysRevSTAB.18.063401

\section{INTRODUCTION}

Radio-frequency (rf) photoinjectors [1] are the most adequate instruments to produce high brightness electron beams of energies up to several $\mathrm{MeV}$. Rf photoinjectors have various applications, mainly as drivers for future linear colliders and especially X-ray free-electron laser (FEL) facilities, but also as direct sources for electron diffraction experiments $[2,3]$ or in compact gamma-ray or $\mathrm{X}$-ray sources based on inverse Compton scattering [4,5].

$\mathrm{X}$-ray FELs are modern research tools in multiple fields of science such as biology, physics, chemistry, medicine, and material science. Most of the current and future planned FELs, including SwissFEL presently under construction at the Paul Scherrer Institute [6], employ laserdriven rf injectors.

The electron beam emittance is a key parameter for FELs. To produce diffraction-limited transverse-coherent radiation, the electron transverse emittance should be $\varepsilon_{n} / \gamma \approx \lambda / 4 \pi$ for an efficient electron-radiation interaction, where $\varepsilon_{n}$ is the normalized beam emittance, $\gamma$ is the Lorentz factor and $\lambda$ is the FEL radiation wavelength. This condition indicates that by reducing the normalized emittance the required final beam energy can be decreased, which allows a shorter and more economical accelerator. Moreover, for a given beam energy, if the bunch has a smaller emittance, the FEL radiation power will be larger and the undulator beam line to achieve saturation will be

*eduard.prat@psi.ch

Published by the American Physical Society under the terms of the Creative Commons Attribution 3.0 License. Further distribution of this work must maintain attribution to the author $(s)$ and the published article's title, journal citation, and DOI.
PACS numbers: 29.25.Bx, 29.27.Fh, 41.60.Cr, 52.59.Wd

shorter. For instance, for the $200 \mathrm{pC}$ charge operation mode of SwissFEL, if the emittance is reduced from the nominal value of $0.43 \mu \mathrm{m}$ to $0.25 \mu \mathrm{m}$, the FEL power increases about three times [7].

The emittance of the cathode or intrinsic emittance has a strong influence on the final emittance of the electron beam: for example, numerical simulations done for the $200 \mathrm{pC}$ case of SwissFEL show that the contribution of the intrinsic emittance to the final emittance is around 70\% [8]. The intrinsic emittance depends on the initial kinetic energy of the electron beam emitted from the surface of the cathode and it can be formulated as [9]

$$
\varepsilon_{\text {int }}=\sigma_{l} \sqrt{\frac{\phi_{l}-\phi_{\mathrm{eff}}}{3 m_{e} c^{2}}},
$$

where $\sigma_{l}$ is the rms laser beam size, $\phi_{l}$ is the laser photon energy, $\phi_{\text {eff }}$ is the effective work function of the photocathode material, and $m_{e} c^{2}$ is the electron's rest mass energy. We call $\varepsilon_{\text {int }} / \sigma_{l}$ the normalized intrinsic emittance, since it is independent of the laser beam size. We note that the above expression is correct if tilted-surface effects related to the surface roughness of the cathode are negligible [10].

The effective work function of a metal photocathode $\phi_{\text {eff }}$ is obtained by subtracting the Schottky effect $\phi_{\mathrm{Sch}}$ from the material work function $\phi_{w}$ [11]:

$$
\phi_{\mathrm{eff}}=\phi_{w}-\phi_{\mathrm{Sch}}=\phi_{w}-\sqrt{\frac{e^{3}}{4 \pi \varepsilon_{0}} \beta E_{c}(\varphi)},
$$

where $e$ is the charge of the electron, $\varepsilon_{0}$ is the vacuum permittivity, $\beta$ is the local field enhancement factor, which 
includes the effects related to the cathode surface properties, and $E_{c}(\varphi)$ is the applied field on the cathode at the extraction phase $\varphi$. For a given photocathode, the normalized intrinsic emittance can be reduced either by decreasing the laser photon energy or by reducing the rf field at the cathode.

When $E_{c}(\varphi)$ varies in a small range, the quantum efficiency (QE) of a metal photocathode depends quadratically on the difference between the laser photon energy and the effective work function $[9,12]$ :

$$
\mathrm{QE} \propto\left(\phi_{l}-\phi_{\mathrm{eff}}\right)^{2} .
$$

From Eqs. (2) and (3) the effective work function can be obtained from a so-called Schottky scan, i.e., by measuring the emitted charge as a function of the extraction phase $\varphi$.

In the recent past significant efforts have been put into measuring the intrinsic emittance in rf photoinjectors, see for example Refs. [12-15]. The intrinsic emittance dependence on the laser wavelength has been verified in a diode gun [16] and recently also in a rf gun [15]. In Ref. [12] the dependence on the rf field at the cathode was investigated, but normalized intrinsic emittances in the upper range of what is expected from theory were obtained (in the order of $900 \mathrm{~nm} / \mathrm{mm}$ ). Here we present measurements of the intrinsic emittance as a function of the field at the cathode that confirm the theoretical expectations expressed in Eqs. (1)-(2). This has been possible thanks to our accurate and high-resolution method to determine the emittance of very low charge density beams, which are not affected by space-charge effects-a precondition to measure the intrinsic emittance. In addition to the emittance measurements, we have reconstructed the normalized intrinsic emittance by performing Schottky scans.

The measurements have been done at the SwissFEL Injector Test Facility [17], which is introduced in Sec. II. After outlining our measurement procedures in Sec. III, we present the measured intrinsic emittance values in Sec. IV, discuss the results in Sec. V and give our conclusions in Sec. VI.

\section{THE SWISSFEL INJECTOR TEST FACILITY}

The SwissFEL Injector Test Facility [17] was the main test bed and demonstration plant for the SwissFEL project [6]. SwissFEL is presently under construction with first hard-X-ray FEL radiation expected by 2017. The SwissFEL Injector Test Facility was in operation between 2010 and 2014.

The electron bunches were generated in an S-band $\mathrm{rf}$ photoinjector gun operated at $10 \mathrm{~Hz}$ repetition rate. The measurements were done with a compact turn-key Nd:YLF laser with a fixed wavelength of $262 \mathrm{~nm}$ and a Gaussian temporal profile with a rms pulse length of $2.5 \mathrm{ps}$. The 2.6cells rf gun, originally developed for high-current operation at the CLIC Test Facility at CERN [18], gives a nominal field at the cathode at the injection phase of $49.9 \mathrm{MV} / \mathrm{m}$, which corresponds to a total beam energy at the gun exit of $7.1 \mathrm{MeV}$.

Four S-band accelerating structures accelerated the beam up to its design energy of $250 \mathrm{MeV}$. After that an X-band linearizing cavity and a magnetic chicane were used to compress the beam longitudinally. For the measurements presented here, the gun phase was set to minimize the energy spread of the beam by using a spectrometer after the gun, the S-band structures were operated at on-crest acceleration, and the X-band cavity was switched off. Further downstream of the chicane a dedicated diagnostics section allowed us to characterize the accelerated beam: an S-band transverse deflecting cavity, several quadrupole magnets and beam screens measuring the transverse beam profile were available for projected and slice emittance measurements. More detailed information on the SwissFEL Injector Test Facility can be found in Ref. [17].

The measurements presented here were performed with a cathode made of pure copper. The cathode surface was diamond turned to an average roughness of a few nanometers [19]. We applied a careful cleaning of the cathode which consists of ultrasound treatment and an annealing cycle under vacuum at $250^{\circ} \mathrm{C}$ for 10 hours.

\section{MEASUREMENT PROCEDURES}

The final emittance of the rf photoinjector is determined by the intrinsic emittance and by the contributions from space-charge and rf-field effects. ${ }^{1}$ The rf gun phase is normally set to minimize the energy spread of the electron beam and therefore its final emittance. Even in this case, however, the focusing and thus the optics varies for different individual slices along the bunch because of the rf field variation seen by the beam (in the SwissFEL case the bunch can cover up to about 10 degrees of the gun rf period). Due to the optics mismatch along the bunch the emittance of the whole beam, i.e., the projected emittance, is larger than the emittance of a single slice of the bunch with approximately constant energy and optics. The measurement of the slice emittance gives direct access to the intrinsic emittance, provided that the influences of space charge forces and rf field inhomogeneities (e.g., quadrupolar field components as discussed below) on individual slices are negligible. In our measurements we divide the beam into ten slices per rms bunch length and average the emittance over seven slices around the longitudinal beam center to obtain the so-called core slice emittance. The errors of our core slice emittance measurement take into account the variation of the emittance along the slices and the statistical fluctuations between individual emittance measurements.

\footnotetext{
${ }^{1}$ By rf-field effects we mean all rf contributions to the final emittance except the Schottky effect, which is included in the definition of the intrinsic emittance [see Eq. (2)].
} 
A reliable and high-resolution method is crucial for the precise measurement of the slice emittance. We determine the core slice emittance at the high energy section of the accelerator by streaking the bunch vertically with the rf transverse deflector and measuring it on a high-resolution and high-sensitivity yttrium-aluminum-garnet (YAG) screen. The YAG screen is observed under an innovative imaging geometry that fulfills the Snell-Descartes law of refraction and the Scheimpflug condition at the same time [20]. This is achieved by placing the scintillator at an angle of 8.1 degrees with respect to the electron beam, which allows reaching a measured beam size resolution of $15 \mu \mathrm{m}$-much smaller than the crystal thickness of $100 \mu \mathrm{m}$. The pixel size of the profile monitor was calibrated with an ISO12233 resolution target of known dimensions. To determine the slice emittance we measure the slice beam size for different optics transformations. The slice beam sizes are obtained from Gauss fits to the full horizontal profiles of the longitudinal slices of the bunch. For our beams the application of Gauss fits gives equivalent results as the rms method, while being faster and more robust [7].

Five quadrupole magnets are used to vary the optics for the horizontal slice emittance measurements. This is done in a systematic way to scan the phase advance in the horizontal plane, to set a fixed vertical phase advance between the deflector and the profile monitor close to 90 degrees for an optimal longitudinal resolution, to have a large horizontal $\beta$-function (between $35 \mathrm{~m}$ and $40 \mathrm{~m}$ ) at the screen for better emittance resolution, and to keep a small vertical $\beta$-function (smaller than $10 \mathrm{~m}$ ) to limit the streaked beam size on the profile monitor. For a final beam energy of $250 \mathrm{MeV}$, the normalized emittance resolution is about $2-3 \mathrm{~nm}$, and the longitudinal resolution is about $13 \mathrm{fs}$. The statistical and systematic errors of the emittance are quantified to be below 5\%. A more detailed description of the slice emittance measurements can be found in Ref. [7].

The YAG screen allows us to reliably measure beam sizes for bunch charges as low as $1 \mathrm{pC}$ and below, where space-charge effects are insignificant for our laser beam sizes. The space-charge limit has been determined by decreasing the bunch charge at fixed laser beam size until the emittance was not further reduced, as shown in Fig. 1.

The normalized intrinsic emittance $\varepsilon_{\text {int }} / \sigma_{l}$ is determined by measuring the core slice emittance as a function of the transverse laser beam size, which is varied by changing the laser aperture. After the space-charge threshold is found, we keep the surface charge density constant during the aperture scan to avoid space-charge effects.

Our gun has noncoaxial if feeds from opposing sides which generate a quadrupole field. Due to this effect, the dependence of the emittance on the laser beam size is not purely linear, as expected from Eq. (1), but has a quadratic contribution. Taking the quadratic component into account the normalized intrinsic emittance is determined as the linear coefficient in a quadratic fit to the measurement data.

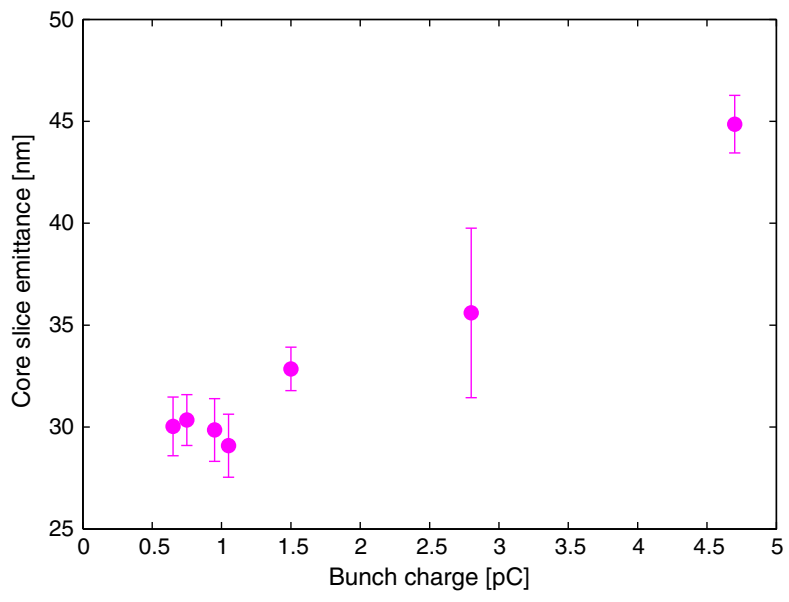

FIG. 1. Example of space-charge limit determination. The field at the cathode was at its nominal value $(49.9 \mathrm{MV} / \mathrm{m})$ and the laser beam size was around $60 \mu \mathrm{m}$. For bunch charges equal or below $1.0 \mathrm{pC}$ the slice emittance is not affected by space charge.

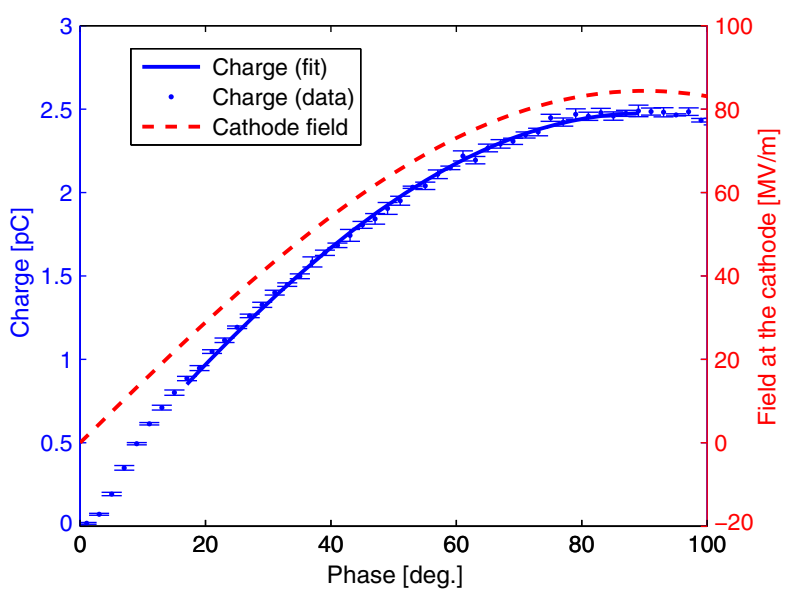

FIG. 2. Example of a Schottky scan: extracted charge (blue points) as a function of the rf phase. The effective work function $\phi_{\text {eff }}$ is obtained by fitting the charge data according to Eqs. (2) and (3). The fit is indicated with the blue line. The red dashed line shows the field at the cathode for the different phases. For this example the cathode field at the extraction phase was at its nominal value $(49.9 \mathrm{MV} / \mathrm{m})$ and the $\mathrm{rms}$ laser beam size was around $60 \mu \mathrm{m}$.

For the Schottky scans, the charge is measured at a calibrated beam position monitor $2.6 \mathrm{~m}$ downstream of the rf gun. The uncertainties of the charge measurement are below the few-percent level. Figure 2 shows an example of a Schottky-scan measurement.

\section{MEASUREMENT RESULTS}

We have measured the normalized intrinsic emittance for three different gun gradients, corresponding to the following fields on the cathode $E_{c}(\varphi): 49.9 \mathrm{MV} / \mathrm{m}$ (nominal value), $34.8 \mathrm{MV} / \mathrm{m}$ and $16.4 \mathrm{MV} / \mathrm{m}$. The total beam 


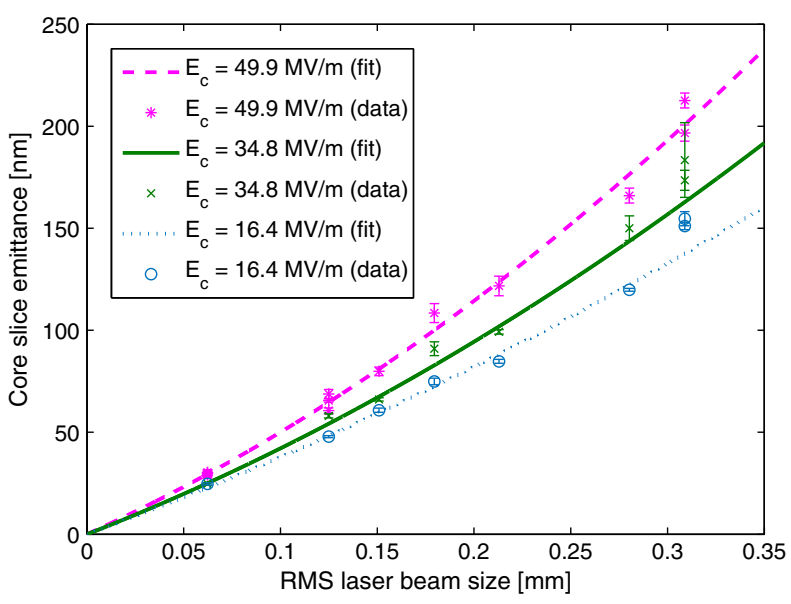

FIG. 3. Core slice emittance as a function of beam size for three different fields on the cathode. The nonlinear effect was reduced for lower fields and the normalized intrinsic emittance varied as expected from theory.

energy after the gun for the different gradients was 7.1 MeV, 5.1 MeV, and 3.7 MeV, respectively. The measurements with the two larger fields were done during the same day and the measurement at the lowest gradient was performed two days later. The laser and accelerator conditions were the same for all measurements, apart from the rf phase adjustments described above.

Figure 3 shows the aperture scan measurements for the three fields at the cathode. The expected beam size along the gun varies between $\approx 50 \mu \mathrm{m}$ and $\approx 80 \mu \mathrm{m}$ for the smallest laser aperture, and between $\approx 300 \mu \mathrm{m}$ and $\approx 400 \mu \mathrm{m}$ for the largest aperture. To avoid space-charge effects, the beam charge density was kept constant at a value below the space-charge limit. The latter was determined individually for each aperture scan as explained

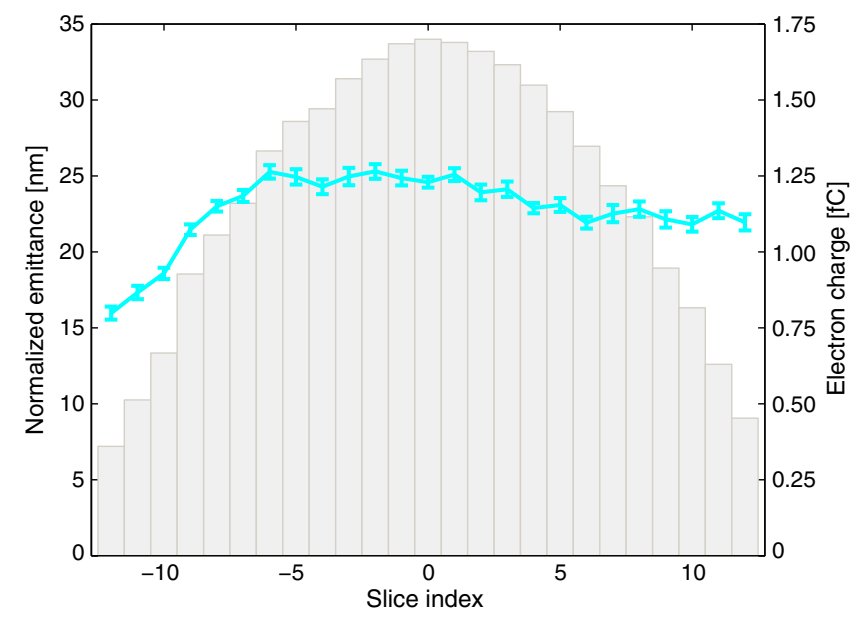

FIG. 4. Normalized slice emittance (cyan, left axes) for a total bunch charge of $30 \mathrm{fC}$. The error bars are obtained by error propagation of the statistical beam-size errors. The longitudinal bunch charge profile is shown as gray bars (right axes).
TABLE I. Reconstructed rf quadratic components and normalized intrinsic emittances as a function of the cathode field at the SwissFEL Injector Test Facility.

\begin{tabular}{lcc}
\hline \hline $\begin{array}{l}\text { Cathode field } \\
\text { MV/m }\end{array}$ & $\begin{array}{c}\text { Quadratic component } \\
\mathrm{nm} / \mathrm{mm}^{2}\end{array}$ & $\begin{array}{c}\varepsilon_{\text {int }} / \sigma_{l} \\
\mathrm{~nm} / \mathrm{mm}\end{array}$ \\
\hline 49.9 & $724 \pm 84$ & $428 \pm 16$ \\
34.8 & $505 \pm 137$ & $370 \pm 25$ \\
16.4 & $321 \pm 105$ & $346 \pm 25$ \\
\hline \hline
\end{tabular}

earlier. The total bunch charge ranged between $0.72 \mathrm{pC}$ and $33.46 \mathrm{pC}$ for the nominal gradient, between $0.15 \mathrm{pC}$ and $4.98 \mathrm{pC}$ for the intermediate gradient, and between $0.03 \mathrm{pC}$ and $1.50 \mathrm{pC}$ for the lowest gradient. Therefore the smallest bunch charge measured, at the lowest gradient and at the smallest laser aperture, corresponding to about $60 \mu \mathrm{m} \mathrm{rms}$ laser beam size at the cathode, was just $30 \mathrm{fC}$. A slice emittance measurement at this ultralow charge was possible thanks to the high sensitivity of our profile monitor and due to the smaller amount of dark current produced by the gun with the lowest $\mathrm{rf}$ gradient. The measurement is shown in Fig. 4. The core slice emittance was below $25 \mathrm{~nm}$.

Table I displays the rf quadratic component and the normalized intrinsic emittance (linear term) for each aperture scan. For the lowest gradient the normalized intrinsic emittance is about $20 \%$ smaller than for the nominal gradient. As expected from the rf-induced effect, the quadratic component decreases approximately linearly with the cathode field. For the nominal gradient, the measured quadratic component can be reproduced with numerical simulations using ASTRA [21] assuming a total quadrupole field in the gun of $0.07 \mathrm{~T}$. This value is consistent with preliminary estimations obtained from the field map of the gun. ${ }^{2}$

We performed Schottky scans for the highest and lowest rf gradients used in the direct emittance measurements (49.9 MV/m and 16.4 MV/m). For each case, the scan was done for different laser apertures (see Fig. 2 for an example). The Schottky scans were done on the same respective days and under the same conditions as the emittance measurements. The resulting effective work functions are $4.46 \pm 0.08 \mathrm{eV}$ for the nominal gradient and $4.48 \pm 0.12 \mathrm{eV}$ for the reduced gradient. This corresponds [see Eq. (1)] to a normalized intrinsic emittance of $421 \pm 62 \mathrm{~nm} / \mathrm{mm}$ and $406 \pm 96 \mathrm{~nm} / \mathrm{mm}$ for a gradient of $49.9 \mathrm{MV} / \mathrm{m}$ and $16.4 \mathrm{MV} / \mathrm{m}$, respectively. The reconstruction of the intrinsic emittance from the Schottky scans matches with the direct emittance measurements

\footnotetext{
${ }^{2}$ More recently we performed measurements of the normalized intrinsic emittance with a newer rf gun developed at the Paul Scherrer Institute [22], which does not have noncoaxial rf feeds and thus is expected to have a smaller rf quadrupole field. As expected the aperture scans showed that the second-order effects were much less pronounced.
} 
shown in Table I, but such scans are not precise enough to verify the variation of the intrinsic emittance in our range of cathode fields. Schottky scans can be used, however, as a straightforward method to obtain a rough estimate of the normalized intrinsic emittance of a photocathode.

We have fitted the measured normalized intrinsic emittances as a function of the rf gradient using Eqs. (1) and (2). The best fit is obtained for an enhancement factor $\beta=$ $0.79 \pm 0.52$ and a work function $\phi_{w}=4.70 \pm 0.07 \mathrm{eV}$. An enhancement factor $\beta$ around 1 corresponds to excellent cathode surface properties, and a work function of around $4.7 \mathrm{eV}$ matches with the expected values for copper-from the different published values in the literature, the average work function for copper is estimated to be $4.66 \pm 0.51$ [23].

The QE at nominal gradient and extraction phase was around $10^{-4}$. Unfortunately, measurements of the QE for reduced gradients could not be performed. According to Eqs. (2) and (3) with our fitted parameters, the QE is expected to decrease by about $30 \%$ for a gradient of $34.8 \mathrm{MV} / \mathrm{m}$ and about $60 \%$ for the lowest gradient of $16.4 \mathrm{MV} / \mathrm{m}$, while the reduction in emittance is about $10 \%$ and $20 \%$, respectively.

\section{DISCUSSION}

For the range of cathode fields explored here the normalized intrinsic emittance is reduced by about $20 \%$. Reducing the rf field has, however, two disadvantages: (i) A lower rf field implies a smaller QE-in our case the QE degradation is estimated to be about $60 \%$ for an emittance improvement of $20 \%$. This can be compensated with a more powerful laser system. (ii) For a reduced rf field the total energy of the beam at the exit of the photoinjector will also be reduced-in our case the energy drops from 7.1 MeV to 3.7 MeV. This will cause an increase of the final emittance due to stronger space-charge effects. This problem could be addressed by an appropriate reoptimization of the gun design aiming at maintaining the exit energy despite the reduced field at the cathode.

An optimization that takes into account the contributions of the intrinsic emittance, the space charge and the rf field is needed to choose the best configuration in a rf photoinjector. Such an optimization must consider the dependence of the intrinsic emittance on the field at the cathode predicted by theory and demonstrated experimentally in our measurements.

\section{CONCLUSION}

We have measured the dependence of intrinsic emittance on the cathode field for copper in a rf photoinjector. The emittance measurements agree well with theoretical expectations, showing that the intrinsic emittance can be reduced by lowering the rf field. We attribute this agreement to a reliable measurement procedure and to an excellent cathode surface quality.
We have measured normalized intrinsic emittances down to about $350 \mathrm{~nm} / \mathrm{mm}$. This is, to the best of our knowledge, the lowest normalized intrinsic emittance ever measured in a rf photoinjector. The empirical validation of these small values was possible through the combination of our highsensitivity profile monitor and our reliable high-resolution measurement procedure.

\section{ACKNOWLEDGMENTS}

We would like to thank Rasmus Ischebeck and his colleagues of the beam diagnostics group of PSI for the development and installation of the SwissFEL profile monitor employed in our measurements. We acknowledge Paolo Craievich for helping us to understand and quantify the quadrupole field component in the rf gun. Furthermore, we thank the extensive contributions of all the PSI expert groups and the SwissFEL team to the construction and operation of the SwissFEL Injector Test Facility.

[1] J. S. Fraser, R. L. Sheffield, E. R. Gray, and G. W. Rodenz, IEEE Trans. Nucl. Sci. 32, 1791 (1985); for a recent review see, e.g., C. Hernandez-Garcia, P. G. O' Shea, and M. L. Stutzman, Phys. Today 61, 44 (2008).

[2] J. B. Hastings, F. M. Rudakov, D. H. Dowell, J. F. Schmerge, J. D. Cardoza, J. M. Castro, S. M. Gierman, H. Loos, and P. M. Weber, Appl. Phys. Lett. 89, 184109 (2006).

[3] P. Musumeci, J. T. Moody, and C. M. Scoby, Ultramicroscopy 108, 1450 (2008).

[4] S. Boucher, P. Frigola, A. Murokh, M. Ruelas, I. Jovanovic, J. B. Rosenzweig, and G. Travish, Nucl. Instrum. Methods Phys. Res., Sect. A 608, S54 (2009).

[5] W. S. Graves, W. Brown, F. X. Kaertner, and D. E. Moncton, Nucl. Instrum. Methods Phys. Res., Sect. A 608, S103 (2009).

[6] R. Ganter, PSI Report No. 10-04, 2012.

[7] E. Prat, M. Aiba, S. Bettoni, B. Beutner, S. Reiche, and T. Schietinger, Phys. Rev. ST Accel. Beams 17, 104401 (2014).

[8] S. Bettoni et al. (to be published).

[9] D. H. Dowell and J. F. Schmerge, Phys. Rev. ST Accel. Beams 12, 074201 (2009).

[10] D. H. Dowell, in Photocathode Physics for Photoinjectors, Workshop, Ithaca, USA (2012), http://www.classe.cornell .edu/Events/Photocathode2012/WebHome.html.

[11] Z. M. Yusof, M. E. Conde, and W. Gai, Phys. Rev. Lett. 93, 114801 (2004).

[12] H. J. Qian, C. Li, Y. C. Du, L. X. Yan, J. F. Hua, W. H. Huang, and C. X. Tang, Phys. Rev. ST Accel. Beams 15, 040102 (2012).

[13] Y. Ding et al., Phys. Rev. Lett. 102, 254801 (2009).

[14] F. Stephan et al., Phys. Rev. ST Accel. Beams 13, 020704 (2010).

[15] M. C. Divall, E. Prat, S. Bettoni, C. Vicario, A. Trisorio, T. Schietinger, and C.P. Hauri, Phys. Rev. ST Accel. Beams 18, 033401 (2015) 
[16] C. P. Hauri, R. Ganter, F. Le Pimpec, A. Trisorio, C. Ruchert, and H. H. Braun, Phys. Rev. Lett. 104, 234802 (2010).

[17] M. Pedrozzi, PSI Report No. 10-05, 2010.

[18] R. Bossart and M. Dehler, in Proceedings of the 5th European Particle Accelerator Conference, Sitges, Spain, 1996 (IOP, Bristol, 1996), p. 1544.

[19] J. Bossert, R. Ganter, M. Schaer, and T. Schietinger, in Proceedings of the 36th International FreeElectron Laser Conference, Basel, Switzerland, 2014 (JACoW, Geneva, 2015), p. 832.
[20] R. Ischebeck and V. Thominet, Patent No. WO201402969 3A1, 2014.

[21] ASTRA, A Space Charge Tracking Algorithm, http://www .desy.de/ mpyflo/ (1997).

[22] J.-Y. Raguin, M. Bopp, A. Citterio, and A. Scherer, in Proceedings of the 26th International Linear Accelerator Conference, Tel Aviv, Israel, 2012 (JACoW, Geneva, 2012), p. 495.

[23] D. H. Dowell, F. K. King, R. E. Kirby, J. F. Schmerge, and J. M. Smedley, Phys. Rev. ST Accel. Beams 9, 063502 (2006). 